\title{
Atom probe tomography characterisation of a laser diode structure grown by molecular beam epitaxy
}

\author{
Samantha E. Bennett ${ }^{1}$, Tim M. Smeeton ${ }^{2}$, David W. Saxey, ${ }^{3,}$, George D.W. Smith ${ }^{3}$, \\ Stewart E. Hooper ${ }^{2}$, Jonathan Heffernan ${ }^{2}$, Colin J. Humphreys ${ }^{1}$, and Rachel A. Oliver ${ }^{1, \dagger}$ \\ ${ }^{1}$ Department of Materials Science and Metallurgy, University of Cambridge, Pembroke \\ Street, Cambridge, CB2 3QZ, United Kingdom.
}

${ }^{2}$ Sharp Laboratories of Europe Limited, Edmund Halley Road, Oxford Science Park, Oxford, OX4 4GB, United Kingdom.
${ }^{3}$ Department of Materials, University of Oxford, Parks Road, Oxford, OX1 3PH, United Kingdom.

\begin{abstract}
$\underline{\text { Abstract }}$
Atom probe tomography (APT) has been used to achieve three-dimensional characterization of a III-nitride laser diode (LD) structure grown by molecular beam epitaxy (MBE). Four APT data sets have been obtained, with fields of view up to $400 \mathrm{~nm}$ in depth and $120 \mathrm{~nm}$ in diameter. These data sets contain material from the InGaN quantum well (QW) active region, as well as the surrounding $p$ - and $n$-doped waveguide and cladding layers, enabling comprehensive study of the structure and composition of the LD structure. Two regions of the same sample, with different average indium contents $(18 \%$ and $16 \%)$ in the QW region, were studied. The APT data are shown to provide easy access to the $p$-type dopant levels,

\footnotetext{
* Present address: The University of Western Australia (M013), 35 Stirling Highway, CRAWLEY WA 6009, Australia.

${ }^{\dagger}$ To whom correspondence should be addressed. E-mail: rao28@cam.ac.uk
} 
and the composition of a thin AlGaN barrier layer. Next, the distribution of indium within the InGaN QW was analyzed, to assess any possible inhomogeneity of the distribution of indium ("indium clustering"). No evidence for a statistically significant deviation from a random distribution was found, indicating that these MBE-grown InGaN QWs do not require indium clusters for carrier localization. However, the APT data show steps in the QW interfaces, leading to well-width fluctuations, which may act to localize carriers. Additionally, the unexpected presence of a small amount $(\mathrm{x}=0.005)$ of indium in a layer grown intentionally as GaN was revealed. Finally, the same statistical method applied to the QW was used to show that the indium distribution within a thick $\mathrm{InGaN}$ waveguide layer in the $n$-doped region did not show any deviation from randomness. 


\section{Introduction}

Light emitting diodes (LEDs) and laser diodes (LDs) fabricated from gallium nitride (GaN) and the $\operatorname{In}_{\mathrm{x}} \mathrm{Ga}_{1-\mathrm{x}} \mathrm{N}$ and $\mathrm{Al}_{\mathrm{y}} \mathrm{Ga}_{1-\mathrm{y}} \mathrm{N}$ alloys are used in diverse technologies including lighting, televisions, mobile phones, optical storage systems and entertainment centers. The massmarket impact of these devices cannot be questioned. Yet as the technologies leap ahead, scientific understanding of the fundamental material structure of the devices sometimes lags behind, which can inhibit the device development process. Innovations in material characterisation techniques for these devices are therefore very important.

Historically used mainly to analyze metals, atom probe tomography (APT) has emerged as a highly valuable microscopy technique for the characterization of semiconductor structures. ${ }^{1-4}$ APT data are three-dimensional (3D) in nature, collected by a destructive field evaporation process, with the position of each individual atom mapped with nanometer precision and its identity determined by time-of-flight mass spectrometry. ${ }^{5}$ In this study, we use APT to analyze the active region, waveguiding and cladding layers in a III-nitride LD structure grown by molecular beam epitaxy (MBE). III-nitride LD layer structures are relatively complex, typically consisting of a quantum well (QW) active region sandwiched between $n$ type and $p$-type waveguiding layers and then further sandwiched between $n$-type and $p$-type cladding layers. The active, waveguide and cladding layers can all have a critical impact on device performance. Precise knowledge of, and control over, layer structural features such as thickness, composition and nanostructure are essential. 
A challenge for use of APT in this study is that the active, waveguiding and cladding layers extend over at least a micrometer, measured along the growth direction. The regions of most critical interest - those close to the active region - are buried within this range and extend over at least a few hundred nanometers. Acquiring high-quality APT data over such a large field of view and at a precise depth within the layer structure is a major challenge. Development of the local electrode atom probe (LEAP) has made it possible to obtain datasets with a large field of view (in excess of $200 \mathrm{~nm}$ in diameter and $1 \mu \mathrm{m}$ in depth), and with high mass resolution, in hours rather than days. This improvement in collection speed results from pulse repetition rates that are two orders of magnitude faster than with previous instruments, an increase made possible by the local electrode geometry. ${ }^{5}$ Despite these advances in instrumentation, application of APT to device structures in III-nitride materials is in its infancy, with only limited APT studies of full LED structures having been reported. ${ }^{6,7}$ Even in these cases, the critical layers were distributed within a relatively short distance of $\sim 50 \mathrm{~nm}$.

The atom probe analysis in this study was conducted using real LD structures, rather than samples grown especially to facilitate the APT analysis. This approach provides the most accurate information on the true device structure: it avoids the uncertainty resulting from the changes to growth conditions or structure used to make samples especially for APT. The material properties and structural information yielded from the APT data can thus be correlated directly with device performance. Key questions investigated in this study include the magnesium dopant level in the $p$-doped layers, the composition of a nominally $5 \mathrm{~nm}$ $\mathrm{AlGaN}$ barrier layer, and the homogeneity of the indium distribution within the InGaN quantum well. The roughness of the quantum well interfaces, and the composition of a layer 
underlying the QW expected initially to consist of $20 \mathrm{~nm}$ of $\mathrm{GaN}$ were also studied. The APT data were further used to investigate the homogeneity of the indium distribution within a nominally $100 \mathrm{~nm}$ InGaN layer.

The homogeneity of the InGaN QW was a significant focus of the investigations. The InGaN QW is a layer of particular interest in the LD structure, as it serves as the gain medium of the laser. InGaN QW active regions are critical for light output from both LED and LD devices. Common features of these devices are the high densities of threading dislocations (TDs) (typically $10^{6}-10^{8} \mathrm{~cm}^{-2}$ ). The TDs thread through the QW region, having propagated upwards from the GaN layers on which the devices are grown. ${ }^{8}$ As TDs are thought to act as non-radiative recombination centers, ${ }^{9}$ some feature of the QW microstructure is believed to localize the charge carriers, ${ }^{10}$ preventing their diffusion to TDs, where emission would be extinguished.

A possible charge carrier localization mechanism in InGaN QWs was suggested by Narukawa et al., ${ }^{11}$ who observed blotchy strain contrast in QWs using transmission electron microscopy (TEM). The authors suggested that this blotchy contrast was due to "clusters" of higher indium content present in the QW. Such indium clustering would lead to nanometerscale regions of low bandgap material within the QW, which would localize carriers. The indium clustering theory was challenged by two of the current authors, who observed that the action of the electron beam on the specimen during imaging in the TEM could cause a blotchy contrast in images of the QWs. ${ }^{12,13}$ This blotchy contrast might be falsely interpreted as being due to nanometer-scale indium clustering in the original $\mathrm{InGaN}$ layer. 
To exclude the effect of electron beam damage, Galtrey et al. ${ }^{3}$ used APT to study an InGaN multiple quantum well (MQW) sample, reporting that the distribution of indium did not observably deviate from that expected in a random alloy. The work by Galtrey et al. ${ }^{3}$ thus asserted that indium clusters are not necessary for bright light emission from LEDs and LDs. In a connected study, Bennett et al. ${ }^{14}$ used APT to study InGaN MQWs that had been exposed to the electron beam in the TEM prior to APT analysis, to study the effect of such exposure. The data revealed an inhomogeneous indium distribution, in contrast to the control sample that had not been exposed to the electron beam. These findings further supported the electron beam-induced damage effect reported by Smeeton et al., ${ }^{12,13}$ and confirmed that the APT technique is able to detect non-random indium distributions, if they exist. Despite this evidence, controversy remains over the existence of indium clustering in as-grown material. ${ }^{15-17}$ A key goal of the present study was to assess the homogeneity of the InGaN QW active region within a real MBE-grown LD structure to seek any evidence of phase separation.

Finally, we note that MBE is not the typical growth method for LD structures. Although less widely used for III-nitride LD fabrication than metal-organic vapor phase epitaxy (MOVPE), MBE has been proven as a technique for fabrication of InGaN quantum well blue/violet LDs. ${ }^{18,19}$ When compared with MOVPE, MBE offers advantages including reduced consumption of source materials, more precise control over impurity doping, and the ability to grow layers without any carbon-containing precursors - thereby reducing carbon impurity incorporation. The growth conditions used in MOVPE and MBE are very different: for example, many growth temperatures tend to be lower in MBE than MOVPE, so the resulting material properties can differ for devices grown by the two techniques. 


\section{Experiment}

The LD structure wafer was grown using a Veeco V80 MBE system, on a commercially available ultra-low TD density template consisting of $\sim 10 \mu \mathrm{m}$ of GaN grown by MOVPE on a sapphire substrate (Lumilog ULD template). The TD density was nominally less than $8 \times 10^{7} \mathrm{~cm}^{-2}$. The LD layer structure is shown in Fig. 1. Gallium, indium and aluminum were supplied using elemental effusion cells. Elemental silicon was used as the $n$-type dopant and bis-(cyclopentadienyl) magnesium $\left(\mathrm{Cp}_{2} \mathrm{Mg}\right)$ was used as the magnesium source for the $p$-type doping. All of the layers below the $\operatorname{In}_{0.18} \mathrm{Ga}_{0.82} \mathrm{~N}$ QW were grown using ammonia as the source of nitrogen, the ammonia being thermally cracked at the surface of the substrate to release nitrogen for growth (so-called " $\mathrm{NH}_{3}-\mathrm{MBE}$ "). The $\mathrm{QW}$ layer and the subsequent $\mathrm{Al}_{0.2} \mathrm{Ga}_{0.8} \mathrm{~N}$ layer were grown using plasma-excited nitrogen gas as the source of nitrogen; the nitrogen gas $\left(\mathrm{N}_{2}\right)$ was excited using a radio-frequency plasma cell (Veeco Unibulb) before reaching the surface of the substrate (so-called plasma-assisted MBE, or "PAMBE"). The remaining layers were grown using $\mathrm{NH}_{3}-\mathrm{MBE}$. The magnesium dopant level in the $p$-type layers was estimated to be $(2-8) \times 10^{19} \mathrm{~cm}^{-3}$, using secondary ion mass spectrometry (SIMS) measurements carried out on separate calibration samples grown under similar conditions.

The InGaN QWs in the laser structure were optimized for emission of blue/green light. MBE growth was carried out using conditions that intentionally created a small range of temperatures across the wafer $\left( \pm \sim 5^{\circ} \mathrm{C}\right)$. This approach provides an efficient method to understand the role of growth temperature on device and layer properties. We find that only the InGaN layers are significantly affected by this small variation in growth temperature. 
Electroluminescence (EL) measurements were obtained by depositing nickel/gold $p$-type contacts onto the top surface of the wafer, fabricating an indium contact to the $n$-type layers and injecting a current density of $0.45 \mathrm{kAcm}^{-2}$. Two regions of the wafer were selected for APT analysis: region A, from a cooler part of the wafer, which exhibited peak EL intensity at a wavelength of $539 \mathrm{~nm}$, and region $\mathrm{B}$, from a hotter part of the wafer, which exhibited peak EL intensity at a wavelength of $488 \mathrm{~nm}$. The shorter emission wavelength in region $\mathrm{B}$ is a consequence of a lower $\operatorname{InN}$ fraction (x) in the $\operatorname{In}_{\mathrm{x}} \mathrm{Ga}_{1-\mathrm{x}} \mathrm{N}$ quantum wells grown at a slightly higher temperature. This temperature effect occurs due to higher rates of desorption of indium from the growing layer at higher substrate temperatures.

Needle-shaped APT samples were prepared by a liftout and annular milling method, similar to the process described by Thompson et al., ${ }^{20}$ using an FEI Helios Nanolab dual-beam focused ion beam/scanning electron microscope (FIB/SEM) equipped with an Omniprobe Autoprobe 200 micromanipulator. A $5 \mathrm{keV}$ low energy cleaning step was employed to remove FIB-damaged material. APT samples were prepared from both region A and region B.

APT data were obtained using a CAMECA (formerly Imago Scientific Instruments) LEAP 3000X HR ${ }^{\mathrm{TM}}$, equipped with a reflectron. Analysis was conducted under ultra-high vacuum, at a base temperature of $30 \mathrm{~K}$, with the sample held at a standing voltage close to the threshold necessary for field evaporation. Controlled field evaporation was induced using a $532 \mathrm{~nm}$ wavelength laser focused onto the sample tip, and pulsed at $200 \mathrm{kHz}$. The laser spot size was less than $10 \mu \mathrm{m}$, with a pulse duration of approximately $10 \mathrm{ps.} \mathrm{A} \mathrm{laser} \mathrm{pulse} \mathrm{energy}$ of $0.01 \mathrm{~nJ}$ was used, with an evaporation rate of 0.03 ions per pulse. The ion position and 
time-of-flight data were reconstructed into $3 \mathrm{D}$ atomic maps using the CAMECA IVAS software. A constant shank angle was used for the reconstruction, assuming a hemispherical end shape. The reconstructions were optimized to obtain flat layer interfaces, as observed by transmission electron microscopy imaging, and to achieve a match with layer thicknesses measured using high-resolution X-ray diffraction. This method was used to study two samples from region $\mathrm{A}$, and a further two samples from region $\mathrm{B}$.

\section{Results and Discussion}

Data sets of up to 63 million ions were obtained using the LEAP instrument, running from the $p$-type layers, through the active region, and into the $n$-type layers. This proved possible despite the biaxial strain at the various layer interfaces, which could have caused fracture of the APT sample. The layers included in some or all of the four APT data sets are indicated with gray shading in Fig. 1. All four data sets contained the InGaN QW, permitting analysis of the homogeneity of the indium distribution within the active region.

An atom map of one of the region A data sets is shown in Fig. 2, with indium atoms represented by black dots (with only $25 \%$ of indium atoms shown, for clarity), and aluminum atoms represented by gray dots ( $25 \%$ of aluminum atoms shown), with all other atoms omitted. The data set runs from the bottom of the nominally $595 \mathrm{~nm} \mathrm{Al}_{0.045} \mathrm{Ga}_{0.955} \mathrm{~N}$ layer, through to the $\mathrm{AlGaN} / \mathrm{GaN}$ superlattice. Above the $\mathrm{QW}$, the data set contains the full p-type waveguide GaN layer, which was known, from high-resolution X-ray diffraction measurements, to be $235 \mathrm{~nm}$ thick. This reference layer thickness was used to calibrate the parameters of the APT reconstruction, and the same reconstruction parameters were applied to each of the layers within this and the other three data sets. This approach is only valid if 
the evaporation fields of the layers are similar. The voltage plots of these runs show a reasonably steady increase as successive layers of these complex structures are removed, suggesting that this reconstruction approach is a satisfactory approximation in this case.

To exclude material near the surface of the samples that might have been damaged by ion bombardment during FIB sample preparation, a central cylinder of $35 \mathrm{~nm}$ diameter was selected for analysis in each of the four data sets. Layer interfaces were aligned approximately perpendicular to the long axis of the cylinder. The analysis that follows is an illustrative example of what can be done with the data to help understand the device structure. The analysis will be reported proceeding through the layers from top to bottom in the structure, beginning with the $p$-doped layers.

\subsection{Magnesium dopants in the p-doped layers}

The $p$-type dopant density strongly affects the device properties such as the series resistance of the diode. As the compositional sensitivity of APT can reach 10 atomic parts per million $(\mathrm{ppm}),{ }^{1}$ it is suitable for the study of dopants. Unfortunately, due to peak overlap between ${ }^{28} \mathrm{Si}^{+}$and ${ }^{14} \mathrm{~N}_{2}{ }^{+}$, silicon dopants cannot yet readily be studied in any APT analysis of IIINitride materials. In the $p$-doped layers of the LD structure, however, it was possible to observe a peak in the mass spectrum associated with magnesium, free of peak overlap with any other atomic species. The mass spectrum for the atom map shown in Fig. 2 can be seen in Fig. 3.

All four APT data sets contained $p$-doped material from the $235 \mathrm{~nm} \mathrm{GaN} \mathrm{layer,} \mathrm{and} \mathrm{one}$ region A data set contained $\sim 10 \mathrm{~nm}$ of material from the nominally $595 \mathrm{~nm}$ AlGaN layer. In 
order to minimise ion optical aberration effects, APT analysis was conducted on data within a central analysis cylinder $35 \mathrm{~nm}$ in diameter. The magnesium dopant levels found for $p$-type layers of the LD structure are shown in Table I. The dopant levels derived from the APT analysis were found to be in the range of $(1-2) \times 10^{19} \mathrm{~cm}^{-3}$ (approximately $100-200 \mathrm{ppm}$ ), which is slightly lower than the SIMS-estimated values of $(2-8) \times 10^{19} \mathrm{~cm}^{-3}$. However, the APT-measured values have the advantage of being assessed directly from the individual LD structure samples, whereas our SIMS results were sourced from separate samples grown under similar conditions. It is worth noting that in earlier APT measurements of $\mathrm{AlGaN} / \mathrm{GaN}$ superlattices, ${ }^{21}$ measured magnesium dopant concentrations were also found to be lower than the values found by SIMS. ${ }^{22}$ Whether these results are due to a systematic underestimate by APT, a systematic overestimate by SIMS, or neither is a matter for further investigation. 


\subsection{Composition of the nominally $5 \mathrm{~nm}$ AlGaN barrier layer}

The composition of the nominally $5 \mathrm{~nm}$ thick $\mathrm{Al}_{\mathrm{y}} \mathrm{Ga}_{1-\mathrm{y}} \mathrm{N}$ barrier layer immediately above the QW has significant influence on the device properties, but is very difficult to measure precisely using high-resolution X-ray diffraction. An initial estimate of $\mathrm{y}=0.20$ was obtained through analysis of calibration layers grown under similar conditions in simpler layer structures; however, this result does not necessarily transfer to the property of the layer when incorporated into the full LD structure. The APT data were used to obtain a value for the bulk level of aluminum within this layer. In all four data sets, the AlGaN layer was isolated within the central analysis cylinder using an isoconcentration surface set at half the maximum aluminum content of the layer. The APT-measured aluminum content of the thin AlGaN layer for all four data sets is shown in Table II. Note the high degree of consistency of these values between separate samples. With an average aluminum content of $y=0.21 \pm$ 0.01, the APT analysis provides a more precise compositional measurement of the thin AlGaN layer, and also confirms the efficacy of the XRD-based estimate.

\subsection{Homogeneity of the InGaN QW}

The next layer analyzed was the $\operatorname{In}_{\mathrm{x}} \mathrm{Ga}_{1-\mathrm{x}} \mathrm{N}$ QW active region. The maximum indium content of the QW was defined as the highest value on a one-dimensional (1D) concentration profile running vertically through the QW, using a $1 \mathrm{~nm}$ fixed bin width. As has been observed previously by Müller et al., ${ }^{23}$ the concentration of nitrogen was underestimated in our APT data sets, due in part to the formation of neutral $\mathrm{N}_{2}$ (which does not usually trigger a detector signal) during field dissociation of cluster ions. Therefore, the $(\mathrm{In}+\mathrm{Ga}): \mathrm{N}$ stoichiometry was not exactly 50:50 in our data sets, so atomic percent In values were converted to $\mathrm{x}$ using $\mathrm{In} /(\mathrm{In}+\mathrm{Ga})$. As Table III shows, on average, the maximum indium content of the region A quantum well was $\mathrm{x}=0.18 \pm 0.02$. This value was greater than that from region $\mathrm{B}$ 
$(x=0.16 \pm 0.01)$, which emitted at a shorter wavelength. The higher indium content of the QW in region A is consistent with the slightly lower growth temperature, compared with region B. This difference in indium content serves to explain the different emission wavelengths from regions $\mathrm{A}$ and $\mathrm{B}$ of the $\mathrm{LD}$ wafer.

The homogeneity of the indium distribution within the QW active region was analyzed in all four data sets, using $\chi^{2}$ analysis. ${ }^{24}$ This type of statistical analysis, which has been described by Galtrey et al., ${ }^{3}$ was used to determine whether there was any statistically significant deviation from randomness within the QW indium distribution. To ensure that only in-plane variations of the indium content would be considered, a $15 \mathrm{~nm}$ square region of interest was defined near the center of each QW, with a depth of $1 \mathrm{~nm}$ in the $z$-direction. Within these volumes, the data were divided into 50 atom bins. By comparing the observed indium distribution with that expected from a random alloy, $p$ values could be calculated, where the $p$ value indicates the probability that the observed distribution could have occurred by chance in a random alloy. A $p$ value of less than or equal to 0.05 is generally interpreted as justification for rejecting the null hypothesis that the observed distribution is random. All calculated $p$ values exceeded 0.05 , with a mean $p$ value of 0.51 . Hence there was no indication that the indium distribution deviated from that expected in a random alloy for any of the data sets.

A comparison between the observed and expected indium distributions within the QW from a region A data set can be seen in Fig. 4. The observed data suggest that these MBE-grown InGaN QWs have a microstructure similar to the MOVPE-grown wells that have been studied previously by APT, where the InGaN was shown to be a statistically random alloy. ${ }^{3}$ 
Therefore, in these APT-examined samples, indium clustering does not appear to be necessary for bright light emission.

Theoretical modelling, based on APT data, has been employed to understand the nature of the charge carrier localization in the absence of indium clustering for QWs emitting light at

similar wavelengths to the QWs analysed in this paper. ${ }^{25}$ The modelling showed that, for QWs with a statistically random distribution of indium, localisation may occur at regions of higher indium concentration formed inevitably due to the random nature of the alloy material, or at regions of locally increased QW thickness. The next section will focus on the measurement of thickness variations within the QW.

\subsection{InGaN QW interface roughness}

To determine whether carriers might be localized at regions of locally increased QW thickness in our MBE-grown QW, the roughness of the upper and lower interfaces of the QW was investigated. The upper interface was defined as that between the QW and the nominally $5 \mathrm{~nm}$ AlGaN layer, and the lower interface was defined as that between the QW and the nominally $20 \mathrm{~nm} \mathrm{GaN}$ layer. The method used followed that of Galtrey et al., ${ }^{26}$ in which In isoconcentration surfaces with values between $\mathrm{x}=0.02$ and $\mathrm{x}=0.1$ (or approximately $1-5$ at.\% In) were used to define the interfaces. Fig. 5(a) shows the upper interface for a typical data set, with an isoconcentration surface of $x=0.04$. Fig. 5(b) shows the lower interface from the same data set. The lower interface appears to be rougher than the upper one in this data set. The same analysis was conducted with all four data sets, and the mean RMS roughness values were found for both interfaces. To assess the hypothesis that the two means were different, a Student's t-test was applied. This test suggested a confidence level greater 
than $97.5 \%$ that the difference in means is genuine, indicating that the lower interface was rougher, on average, than the upper one.

With MOVPE-grown InGaN QWs, Galtrey et al. ${ }^{26}$ found that the upper interface was statistically rougher, on average, than the lower interface. Galtrey et $a l .{ }^{26}$ reported a mean RMS roughness value of $0.34 \mathrm{~nm}$ for the upper interface, and $0.18 \mathrm{~nm}$ for the lower interface. Here, we find a mean RMS roughness of $0.22 \mathrm{~nm}$ for the upper interface, and $0.35 \mathrm{~nm}$ for the lower interface. Neither the upper or lower interface is quite as smooth as that found by Galtrey et $a .^{26}$ for the lower interface in the MOVPE-grown QWs. Visual inspection of Fig. 5 shows changes in the height of the interface, which we will describe as 'steps'. The steps we observe in the upper interface appear to be roughly monolayer or bilayer in height (where a monolayer is $0.26 \mathrm{~nm}$ ), in common with those observed by Galtrey et al.. ${ }^{26}$ The steps in the lower interface are considerably larger, corresponding to multiple monolayers, with typical step heights of around $1 \mathrm{~nm}$. Galtrey et al. ${ }^{26}$ showed that for their data sets, monolayer and bilayer steps observed by APT were not a consequence of the type of analysis used, or of the diffuseness of the interface, via comparison with model data sets. Hence, the steps observed here are also likely to relate to real, physical roughness of the interfaces.

In considering the roughness of the QW interfaces, one important point to note is that the GaN immediately below the quantum well, which will define the roughness of the lower quantum well interface, was grown by $\mathrm{NH}_{3}-\mathrm{MBE}$, whereas the quantum well itself, and the AlGaN layer immediately above it were both grown by PAMBE. Hence, differences in the growth mode in $\mathrm{NH}_{3}-\mathrm{MBE}$ and $\mathrm{PAMBE}$ may account for the differences between the upper and lower interfaces. There are several further reasons why MBE-grown QWs might demonstrate differences in interface roughness compared to those grown by MOVPE. In 
MOVPE, the growth temperatures are higher, and GaN (the surface roughness of which will define the structure of the lower interface) usually grows in a step-flow mode. ${ }^{26}$ In MBE, lower growth temperatures can lead to growth in an island-nucleation and growth (Frank-vander-Merwe) growth mode, and resultant nanoscale roughening has been observed by scanning tunnelling microscopy. ${ }^{27,}{ }^{28}$ Although the InGaN QW layer is also likely to grow in the Frank-van-der-Merwe mode, the presence of indium can act as a surfactant, ${ }^{29}$ potentially reducing the roughening effect. Nonetheless, the presence of occasional steps in the upper interface of the QW suggests that some roughening does occur, and these steps in the upper interface may contribute to electron localization. Due to the strong piezo-electric fields in strained (0001) InGaN quantum wells, the electrons tend to be localized towards the upper interface and the holes towards the lower interface. ${ }^{25}$ Hence, the steps in the lower interface may have more influence on the holes, which may already be localized at regions of higher indium concentration, formed inevitably due to the random nature of the alloy. ${ }^{25}$ Development of a detailed picture of localization in MBE quantum wells would require further modelling work of the type demonstrated by Watson-Parris et al.. ${ }^{25}$

\subsection{Composition of the nominally $20 \mathrm{~nm}$ GaN layer}

The layer just below the InGaN QW was intended to be grown as a $20 \mathrm{~nm}$ thick GaN layer with the MBE indium source closed, so that no indium incorporation was expected. However, a 1D indium concentration profile through a region A data set indicated that the indium level did not drop to zero within the $20 \mathrm{~nm}$ layer. Fig. 6(a) shows this 1D concentration profile, with the InGaN QW, the nominally $20 \mathrm{~nm}$ GaN layer, and the nominally $100 \mathrm{~nm}$ InGaN layer labelled, for clarity. The dashed line box in Fig. 6(a) indicates the region of the 1D indium concentration profile shown in Fig. 6(b), in which it can 
be seen that the indium level within the $20 \mathrm{~nm}$ layer is at least 0.005 , which is substantially greater than the background level of indium observed in the AlGaN layer above the QW.

The unintended indium present in this layer most likely arises from a gradual incorporation of an excess of indium, which accumulated on the growth surface during the growth of the thick InGaN layer below. Such gradual incorporation of the excess surface indium would serve to explain why the indium concentration falls off slowly, compared to the much sharper InGaNon-GaN interface below. Additionally, both interfaces of the QW are much sharper than the upper interface of the $100 \mathrm{~nm}$ InGaN layer, supporting the suggestion that $\mathrm{In}$ accumulation during the extended growth of the thick layer is an important factor here. (However, in considering this comparison, it must be noted that the $100 \mathrm{~nm}$ InGaN layer, and the material immediately above it were grown by $\mathrm{NH}_{3}-\mathrm{MBE}$, whereas the $\mathrm{QW}$ and the layer immediately above that were grown by PAMBE). The level of indium within the $20 \mathrm{~nm}$ layer was quantified using a region of interest within the central analysis cylinder of $15 \mathrm{~nm}$ in length and $35 \mathrm{~nm}$ in diameter, positioned roughly in the middle of the layer. Table IV shows the indium content of this layer in all four data sets.

\subsection{Homogeneity of the nominally $100 \mathrm{~nm}$ InGaN layer}

The final layer studied within the APT data sets was that grown to be $100 \mathrm{~nm}$ of $\operatorname{In}_{0.02} \mathrm{Ga}_{0.98} \mathrm{~N}$, where the composition was measured by high-resolution X-ray diffraction. The APT data revealed that this layer had an average indium content of $x=0.026 \pm 0.001$, close to the $X$ ray diffraction-measured value. The data from the thick InGaN layer were then analyzed to seek any evidence of phase separation, which would be indicated by a statistically significant deviation from a random alloy distribution. Phase separation at such low indium contents would not be expected from a model based on a spinodal phase diagram, such as the one 
calculated by Ho and Stringfellow. ${ }^{30}$ Nonetheless, Kachkanov et al. ${ }^{31}$ suggested that a nonrandom indium distribution was more likely to be found in low indium content layers than in those with high indium contents.

Again, $\chi^{2}$ analysis was used. A cylindrical region of interest, $20 \mathrm{~nm}$ in diameter and $50 \mathrm{~nm}$ in length, was positioned centrally within the layer in each of the four APT data sets. $\chi^{2}$ analysis with 100 atom bins yielded no indication of a deviation from randomness for the indium distribution for any of the data sets, with all $p$ values in excess of 0.15 . Fig. 7 shows the comparison between observed and expected indium distributions for the nominally $100 \mathrm{~nm}$ InGaN layer from a region B data set, which resulted in a $p$ value of 0.45 . Bin sizes were varied from 50 to 200 atoms, with all sizes yielding similar results. These findings indicate that there is no evidence for phase separation in this very low indium content material, as predicted by phase diagrams of the $\operatorname{InGaN}$ alloy, ${ }^{32}$ and in contrast to the assertions of Kachkanov et al.. ${ }^{31}$

\section{Summary}

An MBE-grown LD structure was examined using atom probe tomography (APT). Four data sets were obtained, two from an area with a green emission wavelength, and two from an area with a blue-green emission wavelength. The data sets were very large, containing volumes of up to $400 \mathrm{~nm}$ in depth and $120 \mathrm{~nm}$ in width, including the active region, as well as the surrounding $p$ - and $n$-type layers. With each APT data set, it was possible to conduct a detailed study of the individual layers in the LD structure. First, the magnesium dopant level was quantified in the $p$-type layers and found to be slightly lower than the SIMS-estimated values. Second, the aluminum content of the very thin AlGaN layer was found to be close to 
the intended value. The question of phase separation in the InGaN QW was addressed using statistical analysis. No indication of a deviation from randomness for the indium distribution was found, which strongly suggests that indium clustering is not required for bright light emission from devices based on MBE-grown InGaN QWs. The interface roughness of the QW interfaces was then analyzed, to investigate possible carrier localization at locally thicker regions of the QW. It was found that there were locally thicker regions, which may act as localization centers, due to a rougher lower interface, compared with the upper interface. Steps of height in excess of $1 \mathrm{~nm}$ were observed in the lower interface. Next, the composition of the layer intended to be grown as $20 \mathrm{~nm}$ of $\mathrm{GaN}$ was investigated. The APT data revealed a non-zero indium level within this layer. This result highlights the need to optimise the growth conditions to prevent the incorporation of residual indium within the GaN layer. Finally, the question of phase separation in the thick InGaN layer was addressed using the same statistical analysis as was applied to the QW. No indication of phase separation was found. This study serves to demonstrate the broad range of topics in the field of semiconductor nanostructures that can be addressed using APT analysis.

\section{Acknowledgements}

This work has been funded, in part, by the EPSRC (GR/S 49391/01). SEB would like to acknowledge funding from Sharp Laboratories of Europe and Pembroke College, Cambridge. RAO would like to acknowledge funding from the Royal Society. We also acknowledge contributions from Valerie Berryman-Bousquet and David Nicholls from Sharp Laboratories of Europe to growth of the sample. SEB also thanks the Institute of Physics for the award of the annual Roy prize for a doctoral thesis on condensed matter and material physics, and the 
Centre for Advanced Structural Ceramics for the award of the Sir Richard Brook prize for a doctoral thesis on ceramics. 


\section{Figure Legends}

Fig. 1. Schematic diagram of the nominal layer structure of the MBE-grown LD structure (not to scale). The layers contained within some or all of the APT data sets are shaded gray. Thicknesses or compositions marked with an asterisk, “*”, were measured by high-resolution X-ray diffraction; all other thicknesses or compositions are nominal values.

Fig. 2. Reconstructed atom map of a region A data set from the MBE-grown LD structure, with $25 \%$ of indium atoms shown as black dots and $25 \%$ of aluminum atoms shown as gray dots (all other atoms omitted). The InGaN QW is near the middle of the data set, as indicated. The nominally $235 \mathrm{~nm} \mathrm{GaN}$ layer used for reconstruction optimization is labeled, as are the other layers contained within the data set.

Fig. 3. Mass spectrum of the atom map shown in fig. 2, showing clear separation of all relevant peaks. The peak associated with the most abundant isotope of magnesium can be identified, in its singly charged state.

Fig. 4. Comparison between the observed distribution of indium within a $\mathrm{QW}$ from region $\mathrm{A}$, and that expected from a random alloy, with 50 atom bins. No statistically significant deviation from randomness was observed. (In a $\chi^{2}$ analysis, a $p$ value of 0.35 was calculated for the data shown). Error bounds are an approximation of the standard error as $\sqrt{ }$, where $n$ is the number of indium atoms collected. 
Fig. 5. The (a) upper and (b) lower interfaces of the QW, displayed with In isoconcentration surfaces of $x=0.04$. The lower interface appears to be rougher than the upper interface, although both interfaces display some visible roughness.

Fig. 6. (a) $1 \mathrm{D}$ concentration profile showing the indium content of a region A data set. (b) Portion of the 1D concentration profile indicated in (a) with a dashed line box. The indium content of the nominally $20 \mathrm{~nm} \mathrm{GaN}$ layer is approximately $\mathrm{x}=0.005$. The approximate positions of the InGaN QW, the nominally $100 \mathrm{~nm}$ InGaN region, and the nominally $20 \mathrm{~nm}$ GaN layer are labelled in both (a) and (b).

Fig. 7. Comparison between observed and expected indium distributions for the nominally $100 \mathrm{~nm}$ InGaN layer from a region B data set, with 100 atom bins. No statistically significant deviation from randomness was observed. (In a $\chi^{2}$ analysis, a $p$ value of 0.45 was calculated for the data shown). Error bounds are an approximation of the standard error as $\sqrt{ } \mathrm{n}$, where $\mathrm{n}$ is the number of indium atoms collected. 
$\underline{\text { Tables }}$

Table I. Magnesium dopant level in the $p$-type layers from all four APT data sets of the LD structure. Error bounds are calculated from an approximation of the standard error as $\sqrt{ } \mathbf{n}$, where $n$ is the number of magnesium atoms collected.

\begin{tabular}{c|c|c|c|c}
\hline $\begin{array}{c}\text { Magnesium dopant } \\
\text { level } / \mathrm{cm}^{-3}\end{array}$ & \multicolumn{2}{|c|}{ Region A data sets } & \multicolumn{2}{c}{ Region B data sets } \\
\hline $595 \mathrm{~nm}$ AlGaN layer & $2.1 \times 10^{19} \pm$ & - & - & - \\
& $0.4 \times 10^{19}$ & & & \\
\hline $235 \mathrm{~nm}$ GaN layer & $1.1 \times 10^{19} \pm$ & $9.7 \times 10^{18} \pm$ & $1.1 \times 10^{19} \pm$ & $1.8 \times 10^{19} \pm$ \\
& $0.06 \times 10^{19}$ & $0.7 \times 10^{18}$ & $0.09 \times 10^{19}$ & $0.09 \times 10^{19}$ \\
\hline
\end{tabular}

Table II. Aluminum content in the layer grown to be $5 \mathrm{~nm}$ of AlGaN, from all four APT data sets of the LD structure.

\begin{tabular}{l|c|c|c|c}
\hline \multirow{2}{*}{} & \multicolumn{2}{|c|}{ Region A data sets } & \multicolumn{2}{c}{ Region B data sets } \\
\hline Aluminum & $0.22 \pm 0.01$ & $0.21 \pm 0.01$ & $0.21 \pm 0.01$ & $0.21 \pm 0.01$ \\
content $/ \mathrm{y}$ & & & & \\
\hline
\end{tabular}

Table III. Average maximum indium content within the $Q W$, for regions $A$ and B of the LD structure.

\begin{tabular}{c|c|c}
\hline & Region A quantum wells & Region B quantum wells \\
\hline Average maximum & $0.18 \pm 0.02$ & $0.16 \pm 0.01$ \\
indium content $/ \mathrm{x}$ & & \\
\hline
\end{tabular}


Table IV. Indium content within the layer intentionally grown as a $20 \mathrm{~nm}$ thick layer of GaN, in all four APT data sets of the LD structure.

\begin{tabular}{c|c|c|c|c}
\hline & \multicolumn{2}{|c|}{ Region A data sets } & \multicolumn{2}{c}{ Region B data sets } \\
\hline Indium & 0.004 & 0.007 & 0.006 & 0.007 \\
content / $x$ & & & & \\
\hline
\end{tabular}




\section{References}

${ }^{1}$ T. F. Kelly, D. J. Larson, K. Thompson, R. L. Alvis, J. H. Bunton, J. D. Olson, and B. P. Gorman, Ann. Rev. Mater. Res. 37, 681 (2007).

${ }^{2}$ K. Inoue, F. Yano, A. Nishida, H. Takamizawa, T. Tsunomura, Y. Nagai, and M. Hasegawa, Ultramicroscopy 109, 1479 (2009).

${ }^{3}$ M. J. Galtrey, R. A. Oliver, M. J. Kappers, C. J. Humphreys, D. J. Stokes, P. H. Clifton, and A. Cerezo, Appl. Phys. Lett. 90, 061903 (2007).

${ }^{4}$ K. Thompson, P. L. Flaitz, P. Ronsheim, D. J. Larson, and T. F. Kelly, Science 317, 1370 (2007).

${ }^{5}$ T. F. Kelly, and M. K. Miller, Rev. Sci. Instrum. 78, 031101 (2007).

${ }^{6}$ G. H. Gu, C. G. Park, and K. B. Nam, Phys. Status Solidi-R 3, 100 (2009).

${ }^{7}$ B. H. Kim, G. H. Gu, and C. G. Park, Mater. Sci. Forum 654, 2370 (2010).

${ }^{8}$ P. Gibart, Rep. Prog. Phys. 67, 667 (2004).

${ }^{9}$ S. J. Rosner, E. C. Carr, M. J. Ludowise, G. Girolami, and H. I. Erikson, Appl. Phys. Lett. 70, 420 (1997).

${ }^{10}$ R. A. Oliver, S. E. Bennett, T. Zhu, D. J. Beesley, M. J. Kappers, D. W. Saxey, A. Cerezo, and C. J. Humphreys, J. Phys. D Appl. Phys. 43, 354003 (2010).

${ }^{11}$ Y. Narukawa, Y. Kawakami, M. Funato, S. Fujita, S. Fujita, and S. Nakamura, Appl. Phys. Lett. 70, 981 (1997). 
${ }^{12}$ T. M. Smeeton, M. J. Kappers, J. S. Barnard, M. E. Vickers, and C. J. Humphreys, Appl. Phys. Lett. 83, 5419 (2003).

${ }^{13}$ T. M. Smeeton, C. J. Humphreys, J. S. Barnard, and M. J. Kappers, J. Mater. Sci. 41, 2729 (2006).

${ }^{14}$ S. E. Bennett, D. W. Saxey, M. J. Kappers, J. S. Barnard, C. J. Humphreys, G. D. W. Smith, and R. A. Oliver, Appl. Phys. Lett. 99, 021906 (2011).

${ }^{15}$ H. Lei, J. Chen, and P. Ruterana, J. Appl. Phys. 108, 103503 (2010).

${ }^{16}$ C. Kisielowski, and T. P. Bartel, Appl. Phys. Lett. 91, 176101 (2007).

${ }^{17}$ T. J. Prosa, P. H. Clifton, H. Zhong, A. Tyagi, R. Shivaraman, S. P. DenBaars, S. Nakamura, and J. S. Speck, Appl. Phys. Lett. 98, 191903 (2011).

${ }^{18}$ S. E. Hooper, M. Kauer, V. Bousquet, K. Johnson, J. M. Barnes, and J. Heffernan, Electron. Lett. 40, 33 (2004).

${ }^{19}$ M. Kauer, S. E. Hooper, V. Bousquet, K. Johnson, C. Zellweger, J. M. Barnes, J. Windle, T. M. Smeeton and J. Heffernan, Electron. Lett. 41, 739 (2005).

${ }^{20}$ K. Thompson, D. Lawrence, D. J. Larson, J. D. Olson, T. F. Kelly, and B. Gorman, Ultramicroscopy 107, 131 (2007).

${ }^{21}$ S. E. Bennett, R. M. Ulfig, P. H. Clifton, M. J. Kappers, J. S. Barnard, C. J. Humphreys, and R. A. Oliver, Ultramicroscopy 111, 207 (2011).

${ }^{22}$ S. E. Bennett, PhD thesis, http://www.dspace.cam.ac.uk/handle/1810/236685, University of Cambridge, (2010). 
${ }^{23}$ M. Müller, B. Gault, G. D. W. Smith, and C. R. M. Grosvenor, J. Phys. Conf. Ser. 326, $012031(2011)$

${ }^{24}$ M. K. Miller, A. Cerezo, M. G. Hetherington, and G. D. W. Smith, Atom Probe Field-ion Microscopy (Oxford University Press, New York, 1996).

${ }^{25}$ D. Watson-Parris, M. J. Godfrey, and P. Dawson, R. A. Oliver, M. J. Galtrey, M. J. Kappers, and C. J. Humphreys, Phys. Rev. B 83, 115321 (2011).

${ }^{26}$ M. J. Galtrey, R. A. Oliver, M. J. Kappers, C. J. Humphreys, P. H. Clifton, D. Larson, D. W. Saxey, and A. Cerezo, J. Appl. Phys. 104, 013524 (2008).

${ }^{27}$ M. H. Xie, S. M. Seutter, W. K. Zhu, L. X. Zheng, Huasheng Wu, and S. Y. Tong, Phys. Rev. Lett. 82, 2749 (1999).

${ }^{28}$ R. A. Oliver, C. Nörenberg, M. G. Martin, A. Crossley, M. R. Castell, and G. A. D. Briggs, Appl. Surf. Sci. 214, 1 (2003).

${ }^{29}$ F. Widmann, B. Daudin, G. Feuillet, N. Pelekanos, and J. L. Rouvière, Appl. Phys. Lett. 73, 2642 (1998).

${ }^{30}$ I.-h. Ho, and G. B. Stringfellow, Appl. Phys. Lett. 69, 2701 (1996).

${ }^{31}$ V. Kachkanov, K. P. O'Donnell, S. Pereira, and R. W. Martin, Philos. Mag. 87, 1999 (2007).

${ }^{32}$ J. Z. Liu, and A. Zunger, Phys. Rev. B 77, 205201 (2008). 
Layer function

Nominal Layer Structure

Dopant

p-type contact layer

$10 \mathrm{~nm} \mathrm{GaN}$

p-type cladding region

$595 \mathrm{~nm} * \mathrm{Al}_{0.045} \mathrm{Ga}_{0.955} \mathrm{~N}^{*}$

$\mathrm{Mg}$

$235 \mathrm{~nm}^{*} \mathrm{GaN}$

waveguide

\&

active region

$5 \mathrm{~nm} \mathrm{Al}_{0.2} \mathrm{Ga}_{0.8} \mathrm{~N}$

$3.5 \mathrm{~nm} \mathrm{In}_{0.18} \mathrm{Ga}_{0.82} \mathrm{~N}$ QW

$20 \mathrm{~nm} \mathrm{GaN}$

$100 \mathrm{~nm} \mathrm{In} \operatorname{lo2}_{0.98} \mathrm{Ga}_{0.98} \mathrm{~N}^{*}$

$55 \mathrm{~nm} \mathrm{GaN}$

n-type cladding region
$480 \times\left(1.6 \mathrm{~nm}^{*} \mathrm{Al}_{0.1} \mathrm{Ga}_{0.9} \mathrm{~N}^{*}+\right.$ $1.6 \mathrm{~nm}^{*} \mathrm{GaN}$ ) superlattice
$220 \mathrm{~nm} \mathrm{GaN}$

$10 \mu \mathrm{m} \mathrm{GaN}$ 


\section{$\mathbf{x}(\mathbf{n m})$}

y $(\mathbf{n m})$

$604020 \quad 0-20-40-60$

In

Al

$-595 \mathrm{~nm}^{*} \mathrm{Al}_{0.045} \mathrm{Ga}_{0.055} \mathrm{~N}^{*}$

$235 \mathrm{~nm}^{*} \mathrm{GaN}$

黄

$5 \mathrm{~nm} \mathrm{Al}_{0.2} \mathrm{Ga}_{0.8} \mathrm{~N}$

$3.5 \mathrm{~nm} \mathrm{In} 0.18 \mathrm{Ga}_{0.82} \mathrm{~N}$ QW $20 \mathrm{~nm} \mathrm{GaN}$

$100 \mathrm{~nm} \mathrm{In}_{0.02} \mathrm{Ga}_{0.98} \mathrm{~N}^{*}$

$55 \mathrm{~nm} \mathrm{GaN}$ 


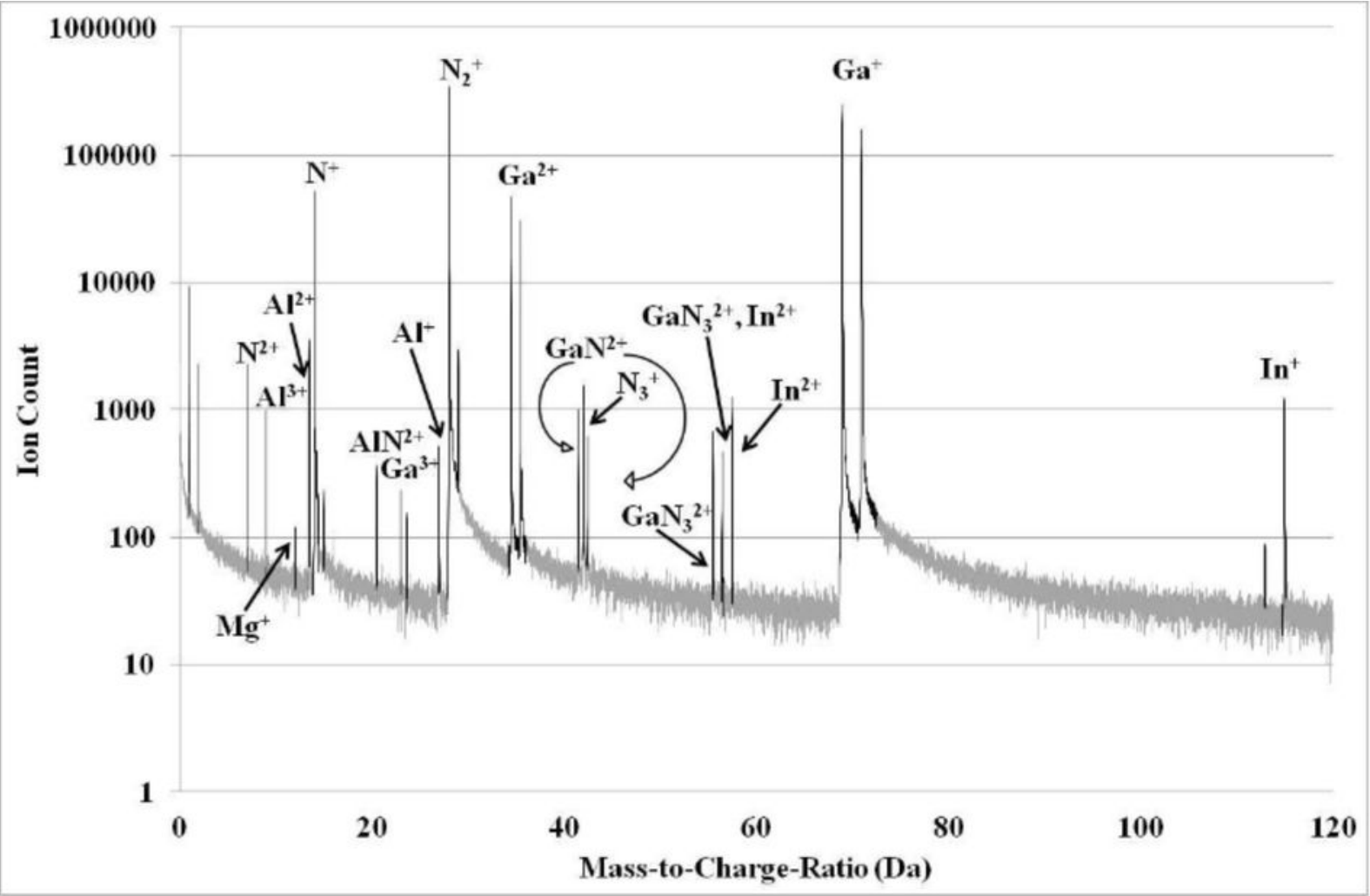


(a)

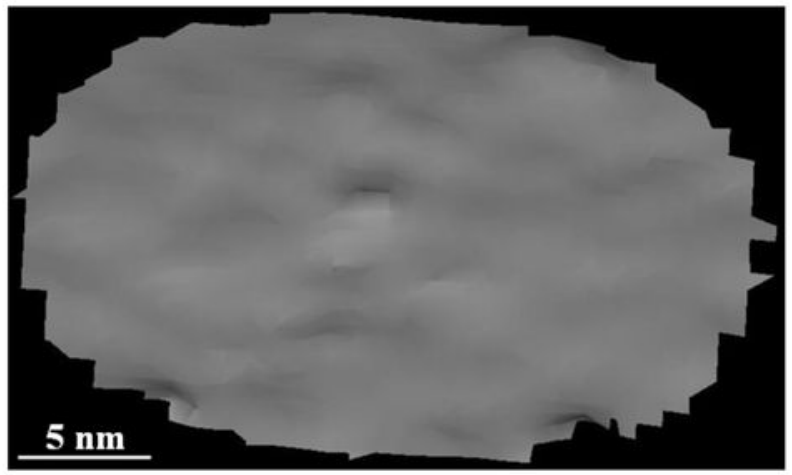

(b)

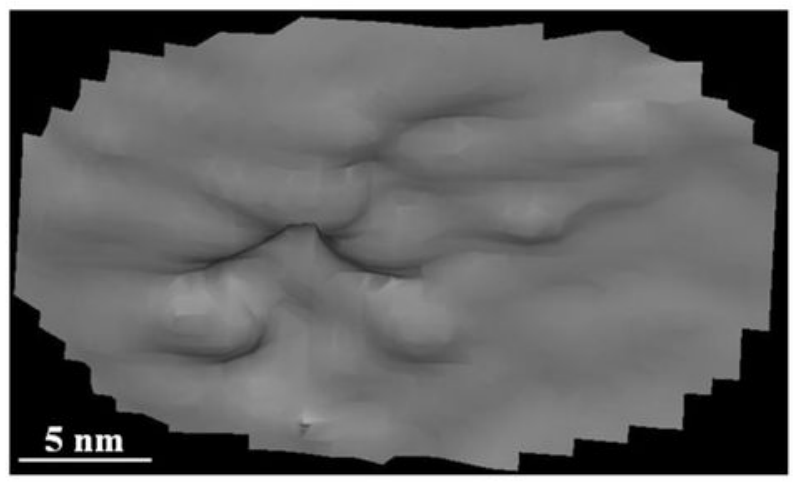


(a)

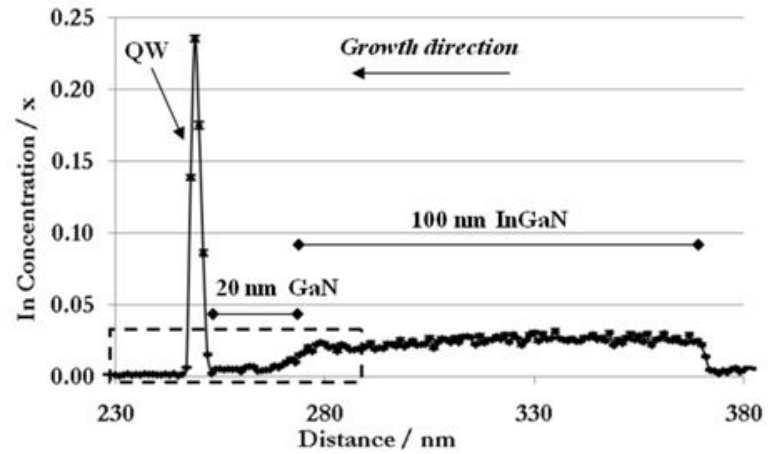

(b)

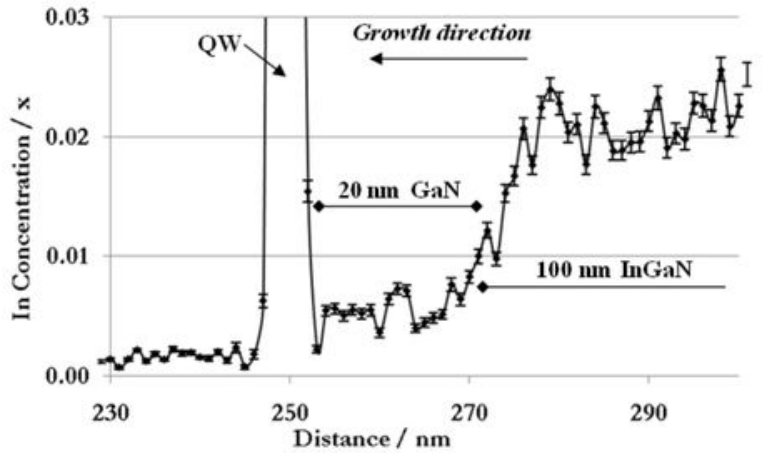




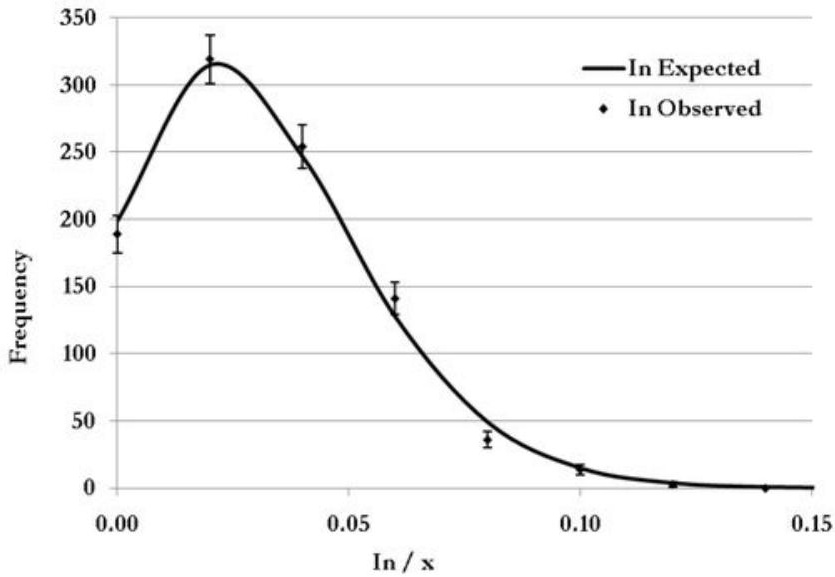

